\title{
Non-Roundabout design of cancel the intersection signal light on horizontal plane
}

\author{
He Yuilin ${ }^{1}$, Andrii Beljatynskij ${ }^{1,}$ and Alexander Ishchenko ${ }^{2}$ \\ ${ }^{1}$ National Aviation University, 1, Cosmonaut Komarov Ave., 03058, Kyiv, Ukraine \\ ${ }^{2}$ Moscow State University of Civil Engineering, 26, Yaroslavskoe shosse, 109377, Moscow, Russia
}

\begin{abstract}
Traffic congestion is a world problem and an important factor restricting urban development. In order to solve the problem of urban traffic congestion, this paper takes the traffic flow theory and the intersection channel design theory as the research foundation, and conducts in-depth research on the causes of congestion at the intersection and the corresponding solutions, and proposes to cancel the traffic lights at the intersection without any stagnation. This paper proposes a new intersection design scheme, which is like the veins of the flower veins to channel the design intersection, cancel the signal light, and the vehicle can pass through the intersection without stagnation. It proposes a new solution to solve the traffic congestion problem. This new design allows the traffic flow to be spatially separated on the horizontal plane, and due to the cancellation of the signal lights, there is no signal waiting at the intersection, and the vehicle can travel without stopping at the intersection. At the same time, this paper also establishes a plane intersection service capability evaluation system based on simulation and quantitative calculation, which provides an evaluation index and proof basis for the non-stagnation driving channel design of the non-roundabout intersection.
\end{abstract}

\section{Introduction}

With the development of social economy, the car holdings of all countries in the world continue to rise (as shown in the chart below), traffic congestion is becoming more and more serious, and even becomes an important reason hindering urban development.In order to solve the traffic congestion problem, scientists from all over the world have worked hard to study the traffic congestion problem, but they have failed to solve the traffic congestion problem in essence.For example: Tan Xiaojie [1], a young transportation scholar in China, published a paper "Research on Knowledge-Based Methodologies and System for Decision Making of Traffic Congesion Management», but she only conducted research on traffic congestion and did not propose An effective solution to the problem of traffic congestion; for example: Zhang $\mathrm{Hu}$ [2], a young transportation scholar in China, who published a paper, «The Mechanism of Congestion and Diffusion of Intersection Groups and Its Control and Induction Collaborative Model», but he only studied the green wave traffic of the port

* Corresponding author: 2580995849@qq.com 
signal in intersection, and he not solved the traffic congestion problem from the root cause. Based on these research backgrounds, this paper uses traffic engineering and transportation management as the theoretical basis to realize the spatial and temporal distribution of traffic flow on the two-dimensional plane by means of channelization. Finally, the signal lights are cancelled, so that the vehicle does not need to wait at the intersection without waiting. Through the intersection, the purpose of solving traffic congestion is achieved.

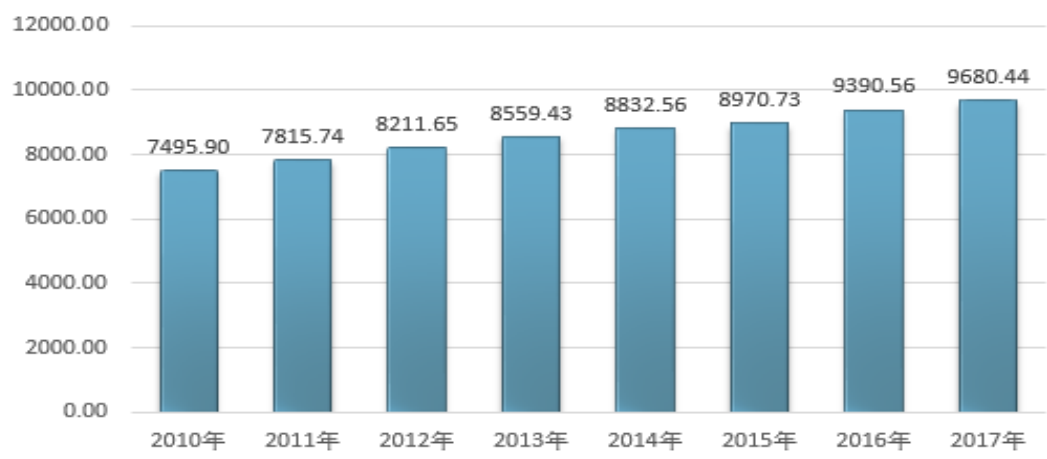

Fig. 1. 2010-2017 global car sales statistics.

The purpose of the canalization design is to regulate the driving of the vehicle, to resolve the conflict between the traffic flows, and to make the road system meet the traffic demand. This design takes the traffic demand as the research basis and aims to solve the traffic congestion problem. The theoretical research and scheme design are carried out. It adopts the principle of traffic flow and the principle of semi-pipelined channelization for intersection planning and design. The dedicated lane here refers to "the separation of traffic factors between motor vehicle and non-motor vehicle lanes, not mixed together" and at the same time, dedicated lanes also refers to the separation of traffic flow in the straight, left and right turn motorways, which become independent individuals, not mixed together, and have special lanes. It also adopts the principle that the conflict points are spatially separated and the convergence points are scattered on the plane to construct the intersection. The conflict points here are spatially separated, which means that the shape of the flower veins is used to establish a semi-over-semi-erected miniature overpass [3]. The lanes of traffic conflicts are dispersed, and then in the form of a single-segment bridge, the traffic that will collide at the intersection is spatially separated up and down; where the junctions are separated on the plane, it means that they will merge in the form of non-signal lights. The lanes are dispersed and then in the form of a channelized approach, the traffic that needs to be merged at the intersection is dispersed on a plane. At the same time, the design is designed to protect pedestrians, separate pedestrians, and human-oriented principles. It is an idealized design for the separation of motor vehicles and non-motor vehicles.

The non-roundabout canalization design of the traffic intersection on horizontal plane is a complete system with strong comprehensive service capability and strong enforceability.It can not only adapt to the complicated and complicated traffic situation, but also fundamentally solve the problem of urban traffic congestion, thus making the commercial [4], economic and cultural exchanges of a region more convenient, making GDP, energy consumption, land use and environmental protectionof for further improvement.

Although the design scheme has strong innovative nature and practical value in theoretical derivation and analysis of ideas, it is lacking in absolute non-separation, minimization of interchangeability, and super-carriage of straight-to-left parallel section. 
Actual problems such as the construction of the half-erected bridge, the width of the road, and the number of lanes need to be improved in conjunction with the specific construction environment.

\section{Materials and Methods}

\subsection{Analysis of traffic characteristics of two-dimensional intersections}

Analysis of operation characteristics of left-turn traffic stream and straight traffic stream.

Whether it is a crossroad or a T-junction, the most conflicting traffic is often the conflict between the left-turn traffic stream and the opposite straight traffic stream, such as a two-way four-lane intersection, a left-turn traffic stream, and a conflict on the opposite straight traffic stream. Up to 16 points [5].

Therefore, the left-turn traffic stream and the straight traffic stream in urban traffic are the main causes of traffic congestion. Only by separating them in time and space can the traffic congestion problem be fundamentally solved.

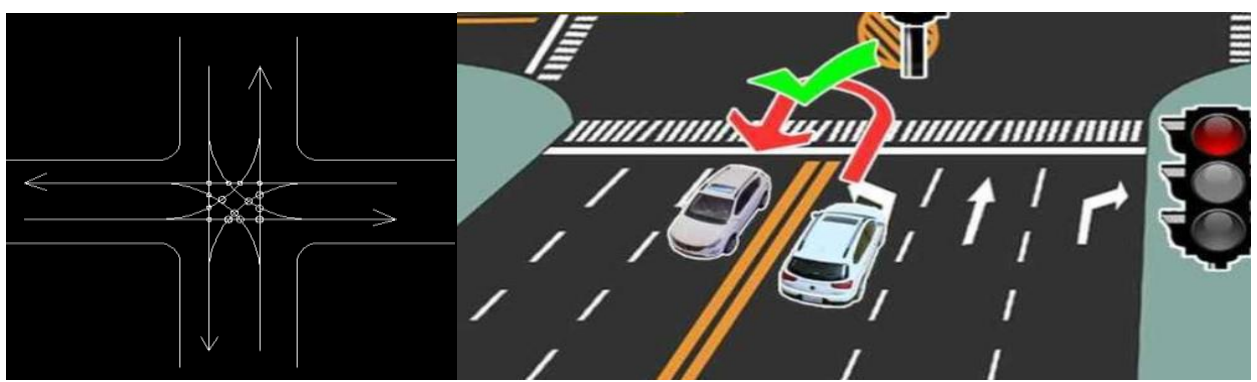

Fig. 2. The left-turn traffic stream and the straight traffic stream conflict point.

Analysis of operation characteristics of left-turn traffic stream and U-turn traffic stream.

In the left-turn and U-turn vehicle operating characteristics of the intersection, the biggest influence on the intersection is the mutual interference between the left turn and the U-turn. For the left-turn and U-turn mixed lanes, the head-to-head distance between the vehicles can be divided into: The headway distance between the left-turning vehicle and the left-turning vehicle, the headway distance between the U-turn vehicle and the U-turn vehicle, and the head-to-head distance between the left-turning vehicle and the U-turning vehicle [6].

By studying the operation characteristics of left-turn traffic stream and U-turn traffic stream, we conclude that the activity space of the U-turn vehicle is smaller than the activity space of the left-turn car. In addition, the number of U-turn vehicles is smaller than the number of left-turn vehicles, so the left-turning vehicle The density is greater than the density of the U-turn vehicle, which creates the complexity of mixing the two traffic flows [7].

After the above analysis, we have obtained the difference in speed, density, and spacing of the vehicle, which often causes mutual interference between the left-turning vehicle and the U-turn vehicle, and sometimes even causes a traffic accident. Therefore, careful analysis of the contradiction between the left-turning vehicle and the U-turn vehicle at the intersection and the determination of an effective solution are important contents of the intersection channelization. Analysis of operation characteristics of left-turn traffic stream and pedestrians stream. 
Right-turn traffic stream is a special traffic stream in the intersection of urban CBD. The particularity is that there is no right-turn signal phase at the intersection. When the right-turning vehicle arrives at the intersection, there is no red light stagnation and it directly leaves the intersection [8].

The speciality of this right-turn traffic stream theoretically realizes the non-stagnation of the traffic stream, but in actual traffic, the right-turn traffic stream of the east-south north-south entrance lane and the non-powered vehicle traffic stream of the east-south north-south entrance lane interfere with each other. There are 8 traffic interference points. The mutual interference between these right-turning vehicles and non-motorized vehicles is the root cause of traffic problems such as pedestrians blocking right-turning vehicles and right-turning vehicles to block non-motorized vehicles.

Therefore, to resolve the contradiction between the right-turning traffic stream and the non-motorized traffic stream and improve the traffic environment at the intersection is one of the important contents to solve the traffic congestion problem.

\subsection{Traffic traffic volume at intersections}

The traffic volume characteristics of the intersection traffic environment are the basis for traffic research at the intersection. Different traffic volumes and distribution characteristics directly affect the characteristics of the intersection and determine the traffic organization of the intersection. This section studies the vehicle arrival characteristics and the spatio-temporal distribution characteristics of the vehicle traffic volume, and hopes to provide a useful theoretical basis for the subsequent channelization design [9].

1) Vehicle arrival characteristics analysis

The arrival characteristics of the vehicle describe the arrival law of the vehicle at the intersection for a certain period of time. The commonly described one is the Poisson distribution formula and the negative binomial distribution formula [10].

Poisson distribution formula:

$$
p(x)=\frac{(\lambda t)^{x} e^{-\lambda t}}{x !},(x=0,1,3 \ldots . .)
$$

$\mathrm{p}(\mathrm{x})$ - The probability of reaching the car during the counting interval.

$\lambda$ - The average number of vehicles coming to the intersection at the unit time.

$\mathrm{t}$ - Count interval time.

e - Mathematical number.

Negative binomial distribution formula:

$$
p(x)=c_{x+\beta-1}^{\beta-1} p^{\beta}(1-p)^{x},(x=1,2,3 \ldots \ldots)
$$

$\mathrm{P}$ and $\beta$ are negative binomial distribution parameters: $0 \leq \mathrm{p} \leq 1$, and $\beta$ is a positive integer.

It can be seen from the above two formulas that when there are too few vehicles on the intersection lane, the vehicles do not interfere with each other, and the traffic environment is excellent, the arrival of the vehicles at the intersection is a random distribution. In this case, the Poisson distribution probability formula should be used. Calculate the arrival probability of the vehicle; when the traffic volume is large and the traffic flow has obvious pulse phenomenon, and the vehicles interfere with each other, the negative quadratic term distribution should be used to estimate the arrival probability of the vehicle. 
2) Analysis of time and space distribution characteristics of traffic volumes

The spatial and temporal distribution characteristics of the traffic volume are the basis of traffic research at the intersection. The different traffic volume arrival and distribution characteristics directly affect the characteristics of the entire intersection and determine the traffic organization of the intersection.

For the influencing factors of the traffic volume at the intersection, the domestic and foreign research scholars believe that the intersection of the intersection, the intersection of the intersection, the entrance guide width of the lane, the entrance mitigation radius, and the width of the loop are all important factors, but in actual traffic. These factors are not obvious. Therefore, this section demonstrates the relationship between the number of intersections, the intersection length of intersections, the radius of the road center and the traffic volume at the intersection by establishing a mathematical model to highlight their importance [11].

Traffic volume factor analysis model of the entrance road:

$$
Q_{w}=m_{1} r_{i}+m_{2} w_{e}+m_{3} n_{e}+m_{4} l_{i}+m_{6} r_{z}+m_{7} q_{e}+b
$$

$\mathrm{Q}_{\mathrm{w}}$ - raffic volume at the intersection,

$\mathrm{r}_{\mathrm{i}}$ - Turning radius,

$\mathrm{W}_{\mathrm{e}}$ - Width of the road at the entrance,

$\mathrm{n}_{\mathrm{e}}$ - Number of lanes at the entrance,

$\mathrm{l}_{\mathrm{i}}$ - Length of intersection,

$\mathrm{r}_{\mathrm{z}}$ - Center island radius,

$\mathrm{q}_{\mathrm{e}}$ - Traffic flow on the ring road,

$\mathrm{b}$ - Correction factor.

\subsection{Analysis of road infrastructure setting conditions at horizontal intersections}

The intersection facilities in the urban CBD area are the premise of good traffic in the urban CBD area. The rationality of their planning determines the service level of the intersection and the operational efficiency of the vehicle. It includes the infrastructure elements of intersection roads such as signal lights, road signs, diversion lines, roadbed curbs, safety islands, fences, parking lines, and road layouts.

Among the road infrastructures at the intersection, the factors that directly determine the driving efficiency of road vehicles are the combination of signal lights and road channelization schemes, because only the complete combination of signal light combination and road channelization scheme can form a systematic traffic organization plan; The characteristics of the interlaced segment, the guiding characteristics of the road entrance, and the characteristics of the safe island are only objective factors that affect the traffic efficiency of the intersection. Therefore, in the canalization of intersections, only the main factors can be grasped and the objective factors can be fully considered to design an canalization scheme that meets the traffic demand.

\subsection{Analysis of service capacity of intersections with traffic lights}

The comprehensive capability of the signalized intersection refers to the comprehensive performance of the signal capacity intersection, the organization index, the safety index, the traffic flow, the driving effect, and the congestion decomposition capability. It is the basis 
for reflecting the value of a signalized intersection, and is also the basis for guiding the construction of a signalized intersection.

Therefore, this section analyzes the traffic commonality, operational efficiency, traffic capacity, safety performance from the intersection of traffic lights, analyzes the inadequacies and merits of signalized intersections, and then, based on these analyses and conclusions, finds the traffic congestion. root cause.

1) Traffic commonality analysis of intersections

Whether it is a T-shaped intersection, a crossroad, or a roundabout, although they each have their own characteristics in the traffic organization, they often have many commonalities in the actual traffic environment, and this section carries out these commonalities here. Listed one by one, hope can serve as a reference for various studies at the intersection.

Traffic commonality of two-dimensional intersections: large traffic flow, high traffic density, low traffic speed, many conflict points and interlacing points, serious mixed interference, high accident rate, dull signal timing, large influence on surrounding environment, uneven time distribution Etc [12].

In the traffic commonality of two-dimensional intersections, the number of vehicles and the distance between the vehicles is caused by the structure of the intersection itself. Because it is the node where the vehicle travels, there are many vehicles and the vehicle spacing is small. However, compared with the middle section of the road, the operation effect is significantly improved, and there are many conflict points and interweaving points, and the traffic accident rate is high because the left-turn, straight-line and U-turn vehicles of the entrances at the intersection cannot be completely separated in time and space. Therefore, there are many conflict points and intertwining points in the flow of the import road, and the traffic accident rate is high.

2) Analysis of traffic stream characteristics of two-dimensional plane signalized intersections.

The intersection of the signal light control is to use the traffic light to guide the vehicle to travel to organize the demand of vehicles in different driving directions at the intersection (time separation method), and use the signal light control method to turn the left turn, the right turn and the straight traffic at the intersection separated in time.

Since the signalized intersection has the disadvantage of periodic cycle, a large number of traffic factors are decelerated, stagnated, and assembled here, and then started and left, thereby greatly losing the running time of the vehicle.

It can be seen from the above that the traffic stream characteristics of the urban CBD signal intersection directly depend on the combination of the intersection signal lights. Once the basic elements such as the total signal time, signal period and phase number of the signal intersection are specified, the driving characteristics of the vehicle at the intersection are clear.

When the phase of the over arrangement signalized intersection, traffic flow characteristics of the vehicle as shown in Fig. 3

When the green signal light is turned on, the vehicle starts to exercise. The vehicle that is stuck in front of the intersection passes through the intersection and then leaves the intersection. The traffic volume quickly reaches the saturation flow rate S. At the end of the green light, when the yellow light is on, the motor vehicle is Stop before the stagnation line line, when the vehicle is in order, the green light is turned on, and the intersecting intersection traffic stream is immediately reduced to the lowest point from the saturated state. 


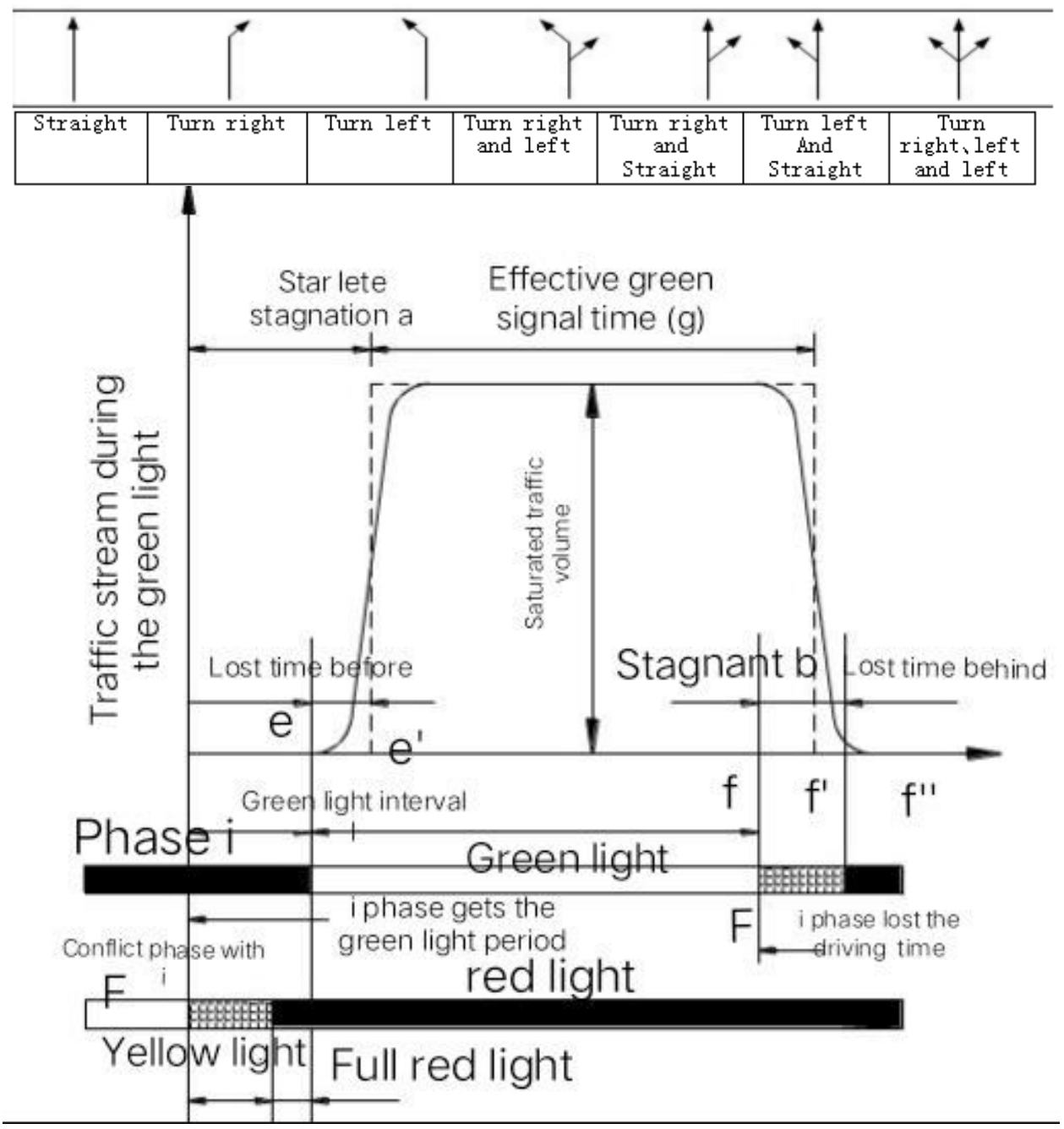

Fig. 3. Traffic stream evacuation at the intersection (green light).

3) Analysis of traffic capacity of two-dimensional plane signalized intersection

The traffic capacity at the intersection refers to the weighted value of the number of vehicles passing through each lane at the intersection, which is an important indicator to measure the traffic capacity of an intersection. The number of vehicles in an entrance lane refers to the maximum number of converted cars passing through the entrance lane of the entrance lane under the conditions of the existing lane width and the length of the adjacent intersection. At present, the methods for calculating the traffic capacity at intersections at home and abroad mainly include: saturated flow rate model, parking line method, conflict point method, CBD road planning standard method, etc., because the road planning standard method in CBD area considers the machine non-mixed line The situation has a strong practical effect on China's traffic research, so this design uses the CBD road planning standard method for analysis and description [13].

The business district road planning standard method is based on the research of the demand for driving vehicles in three directions on each entrance lane of a crossroad, and 
then weights and sums the total number of vehicles in each entrance lane. It is an intersection traffic analysis method.

In combination with the demand characteristics of the motor vehicle at the intersection, the direction of each lane of the entrance lane is divided as follows.
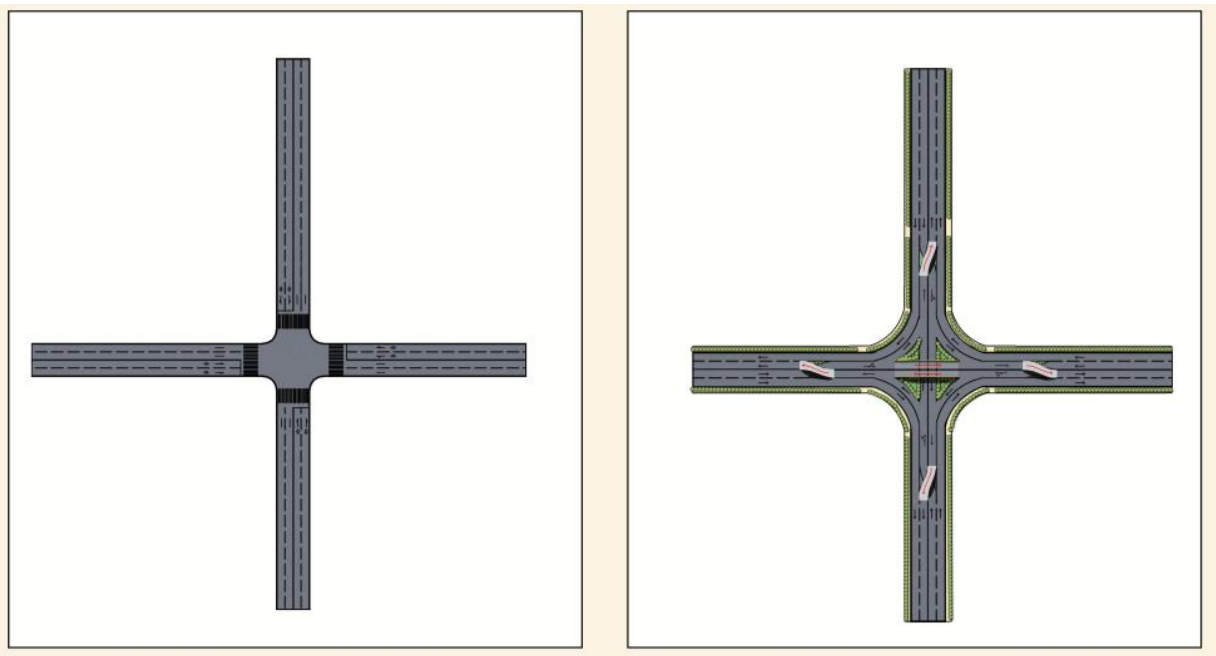

Fig. 4. Motor vehicle lane division.

Calculation formula for traffic capacity of road planning signal intersections in CBD area:

Straight single-lane traffic capacity calculation formula:

$$
c_{i}=\frac{3600}{T}\left(\frac{t_{l}-t_{0}}{t_{i}}+1\right) \varphi
$$

$C_{i}$ - Capacity, T-Signal period, $t_{0}=2.3 \mathrm{~S}$,

$t_{L}$-Green light time, $t_{i}$-Average vehicle distance,

$\varphi$-Correction factor.

Traffic volumes for intersections with specific steering:

$$
C_{I}=\Sigma c_{z} /\left(1-\beta_{l}-\beta_{r}\right)
$$

$C_{I}$ - Traffic volumes at the entrance lane, $\Sigma c_{z}$ - Sum of traffic volumes in straight lane, $\beta_{l}$-Right turn vehicle ratio, $\beta_{r}$ - Left turn vehicle ratio.

Signal traffic volume is one of the important indicators to explore the service capability of CBD signal intersections. This section explains in detail the "CBD Regional Road Planning Standard Method" in the signal intersection traffic volume estimation method. The purpose is to study the CBD. The traffic characteristics of regional intersections provide a basis for research.

Then, this method is used to analyze and study the plane signal intersection. After research and analysis, it is found that although the signalized intersection separates the traffic volume of different flow from time, it solves the problem of contradiction between the intersection point and the interlacing point at the intersection; but it also causes the assembly, queuing and detention of the vehicle at the intersection. Congestion problems, 
especially when the traffic volume exceeds the planned traffic volume, it is easy to cause traffic congestion. Therefore, only by conducting research on this basis can we find a more reasonable urban transportation organization method to meet the demand of traffic factors at the intersection [14].

4) Analysis of Operation Efficiency of two-dimensional Plane Signal Intersection.

The evacuation capacity of the signal intersection is a reference for whether the channelization of the two-dimensional plane intersection and the timing of the traffic lights have been significantly improved, and is an important indicator for judging the traffic capacity of the signalized intersection. In this section, we explain the operation efficiency, delay, horizontal vehicle formation length, and stagnation of the signal intersection, and then calculate the two-dimensional plane intersection based on these factors to find the disadvantage and the need for improvement things of two-dimensional plane intersection.

Average vehicle delay: refers to the sum of time wasted by the motor vehicle for various reasons during the entire vehicle travel. Among these, the waste of time is related to the stagnation caused by the disturbance of the vehicle. This can be calculated by the average vehicle delay at the signalized intersection. The calculation formula is as follows [15]:

$$
d_{I}=\frac{\Sigma d_{A} q_{A}}{\Sigma q_{A}}
$$

$d_{A}$-Lane delay, $\quad q_{A}$ - Correction factor T.V.

The length of the queue of the motor vehicle because of congestion: The motor vehicle leader is an important indicator for judging the congestion at the intersection. It refers to the longitudinal length of the motor vehicle assembly from the parking lane to the entrance guide. The determining factor is the The design of the port signal light in intersection. Specifically, it can be calculated by the formula of the length of the fleet, and the calculation formula is as follows.

$$
L=\left(Q_{1}+Q_{2}+Q_{3}\right) \times l
$$

$l$-The motor vehicle leader, $Q_{1}$-Vehicle that did not pass at the green light, $Q_{2}$-Vehicle arriving at green light, $Q_{3}$-Vehicle that continues after the green light).

Average number of stops: The number of vehicle stops at a two-dimensional plane signal intersection means that the vehicle can pass through the intersection after several stops, and the average number of stops at the signalized intersection refers to the average number of stops when all the vehicles in each lane of the intersection pass through the intersection. . The average number of stops at the intersection is different for each city. The specific value can be obtained by actual investigation of each intersection.

Through the exploration and calculation of the average vehicle delay time, the length of the vehicle congestion queue, and the number of vehicle stagnation, it is found that as long as it is a signalized intersection, there must be traffic stagnation, settling, waiting, etc., and these phenomena are traffic. Direct performance of congestion. Therefore, in order to solve the problem of traffic congestion and realize the smooth running of urban roads, it is necessary to conduct in-depth research on intersections instead of staying at a method of traffic light control.

5) Analysis of safety performance of plane signalized intersection.

The safety factor of a plane intersecting intersection refers to the safety factor between the person at the intersection of the signal and the vehicle, the non-powered vehicle, the driven vehicle and the non-powered vehicle, the traveling vehicle and the traveling vehicle. 
The intersection of cities is the origin of urban traffic problems. Because many transportation needs meet here, traffic problems are serious and vehicles are difficult to drive. However, the safety performance of the plane signalized intersection is difficult to quantify, and the direct evaluation is more difficult. Therefore, this section uses the conflict point and the convergence point indicator between the traffic factors at the intersection for indirect evaluation, because only the flow conflict between different traffic flows and Confluence occupancy will cause traffic accidents such as impacts and scraping between traffic factors.

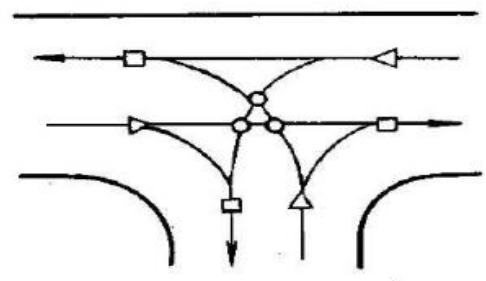

\section{$\mathrm{O}-$ Conflict point, $\square-$ Meeting point}

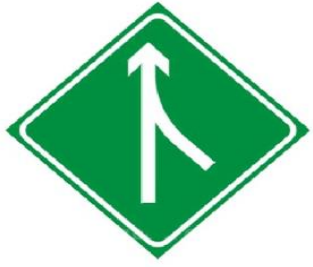

Confluence sign

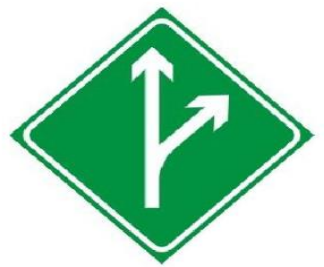

Split sign

Fig, 5. Conflict point

After analysis, it is found that the root cause of high traffic accident rate and low safety performance at the intersection of the signal signal is the mixed driving between the traffic flows at the intersection. Such chaotic driving is not only a chaos between the motor vehicle and the non-motor vehicle, but also a mixed driving of different flow directions between the motor vehicle and the motor vehicle. Therefore, in order to improve the safety performance of the intersections and improve the urban traffic environment, Only by letting the traffic factors at the intersections go their own way can you achieve the desired results.

\section{Originality's and Results}

\subsection{The principle and effect of this design}

Basis and principle of channelization design for two-dimensional intersection

Basis for the intersection of the intersection: vehicles driving at the intersection, if there is no equipment and markings to control the direction of travel, it will destroy the clarity of the traffic flow and reduce the traffic capacity and safety capacity of the intersection. Therefore, in order to drive the vehicle At the intersection, according to the standard marking line, reduce the randomness of traffic, and adopt the intersection channelization method to set signs and markings on the road to regulate the vehicle driving. This is the basis for the intersection channelization. 
In order to increase the safety capability and vehicle driving environment after the intersection intersection planning, the following national basic rules for the two-dimensional intersection intersection channelization should be followed in the channelization treatment:

(a) The lane of the vehicle after the channelization should be clear and easy to distinguish, because the too cumbersome driving scheme is easy to mislead the vehicle, but the safety performance of the intersection is degraded, which makes the vehicle difficult to drive.

(b) Separation and convergence of traffic flow should be avoided. Because of the traffic congestion at the intersection, the vehicle evacuation ability is low because of the conflict points and confluence points at the intersection. Therefore, the conflict point and the junction point of the intersection should be separated as much as possible in the channelization design.

(c) The signs guiding the driving of the vehicle should be conspicuous and clear. Because only clear and striking traffic signs can drive the vehicles in which they are traveling.

(d) The number of lanes at the intersection entrance and the lateral width of the lane should be moderate. Because if the number of lanes at the entrance is too small, it is easy to cause the vehicle to be evacuated at the intersection. If there are too many lanes at the intersection, it is easy to cause the vehicle to select the passage; and if the lane is too narrow, the lateral spacing of the vehicle If it is too small, it will cause parallel danger. If the lane is too wide and the lateral spacing of the lane is too large, it will cause parallel danger.

\subsection{Design principle and Ideas}

Analysis of traffic jams under the control of traffic lights

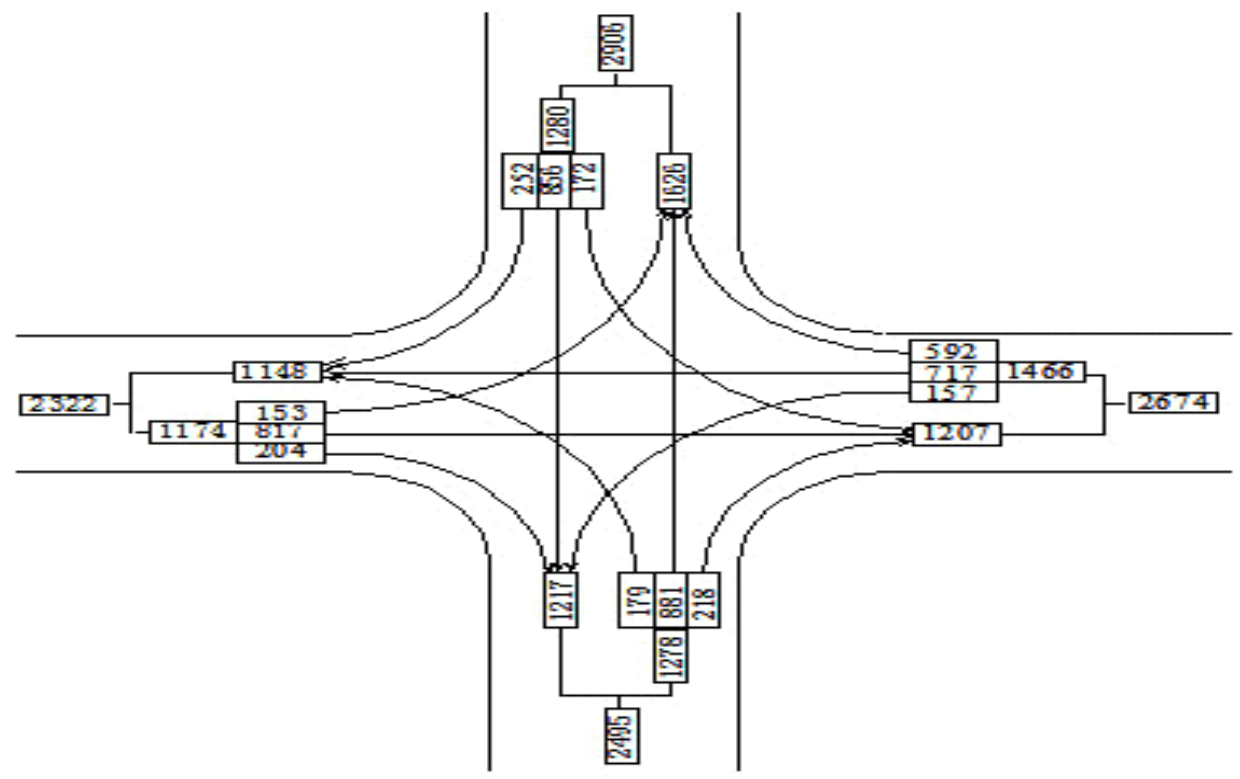

Fig. 6. Scheme of the traffic direction of the traffic intersection. 
Combined Fig.1. In accordance with the point of conflict non-signal transport interchange and the formula for calculating the point of divergence-merging, you can find the conflict point and the number of points of intersection [16]:

$$
\begin{gathered}
\mathrm{Cj}=(\mathrm{nn}(\mathrm{n}-1) *(\mathrm{n}-2)) / 6 \\
\mathrm{Cf}=\mathrm{Cn}=\mathrm{n}(\mathrm{n}-2)
\end{gathered}
$$

Where, $\mathrm{Cj}$ - is the number of conflict points, $\mathrm{Cf}$ - is the number of split points, and $\mathrm{Cn}$-is the number of merge points. Summing up, it turns out an ordinary road with four entrances. At the intersections of the straight line, left and right lanes, there are 16 points of conflict, 8 points of separation and 8 points of confluence [17].

Analysis of the shortcomings of the signalized intersection: According to the control method of the current signalized intersection, it is necessary to set two-way east-west, two-way east-west turn, two-way north-south straight, two-way north-south turn four phases, thus causing the vehicle to reach the intersection You need to wait for three phases (three green lights + three yellow lights + three full red) before you get the right to pass. The section of the special adjacent intersection is too short, and when the green wave cannot be realized, the vehicle is basically enclosed at the adjacent section of the intersection, and only the entrance is not evacuated, thus causing continuous congestion at the intersection. For the peak hours of work and work, the congestion problem is even more unbearable. Analysis of this design analysis: Combined with the above analysis, it can be known that as long as it is a signalized intersection, it will have traffic congestion problems such as vehicle queuing, detention, and waiting. Therefore, only if the vehicle does not stagnate at the intersection, as if it is running smoothly on the road section, the congestion problem can be fundamentally solved. Because this is the only way to ensure that the vehicle entering the intersection is at the intersection of the first time, so that there is no waiting for the vehicle at the intersection, and accordingly there is no traffic congestion problem.

\subsection{Elimination of the traffic light design operation mechanism at the intersection}

The chart is defined as follows: the import route from the south to the north is defined as A import, the import route from west to east is defined as B import, the import route from north to south is regulated as $\mathrm{C}$ import, and the import route from east to west is defined as D import; all right turns are defined as 1, all straight lanes are defined as 2, all left-hand lanes are defined as 3; four non-central interchanges are called white bridges, and the center interchange is called blue bridges.

Provisions for the intersection 2: The motor vehicle and the non-motor vehicle are completely separated; the road infrastructure is good; the weather is good.

On the basis of the above provisions, the operation mechanism of the cross-section cancellation traffic light design scheme is explained as follows:

(a). A1, B1, C1, and D1 follow the indicator head for smooth travel (and no change before channelization).

(b). First (A2, A3), before the first white bridge, drive together on the straight left-supplied road from south to north, and pass under the bridge together, and get ready for diversion before reaching the blue bridge front diversion sign. Drive to the diversion port to divert; then A2 leaves the central intersection from the blue bridge, straight into the second white bridge to pass through the bridge; A3 turn left to follow the sign to the second white 
bridge to pass through the bridge); Finally, the straight, left and right turns of the entire A import are completed.

(c) The driving trajectory of the .C import is exactly the same as the A import; the B and $\mathrm{D}$ imports and the A import pass through the bridge except when crossing the blue bridge, and the other trajectories are identical.

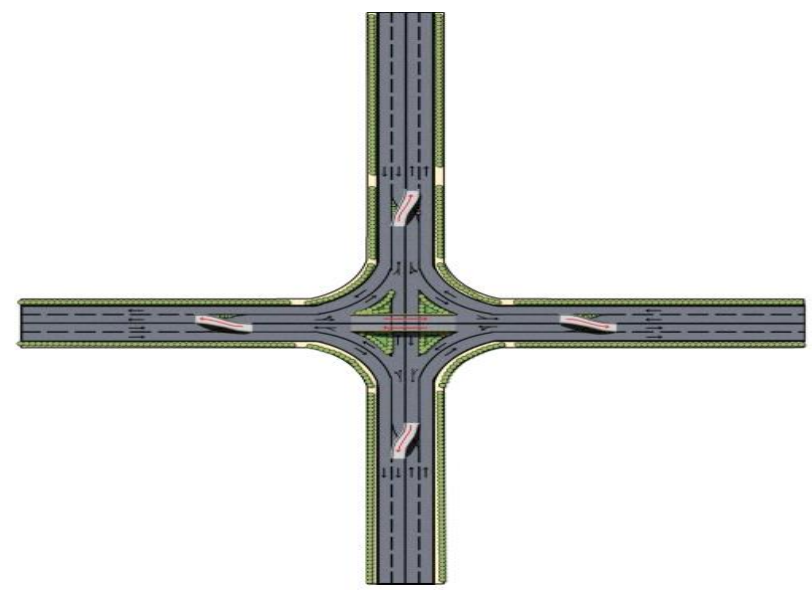

Fig. 7. Cross at the intersection to cancel the traffic light design CAD.

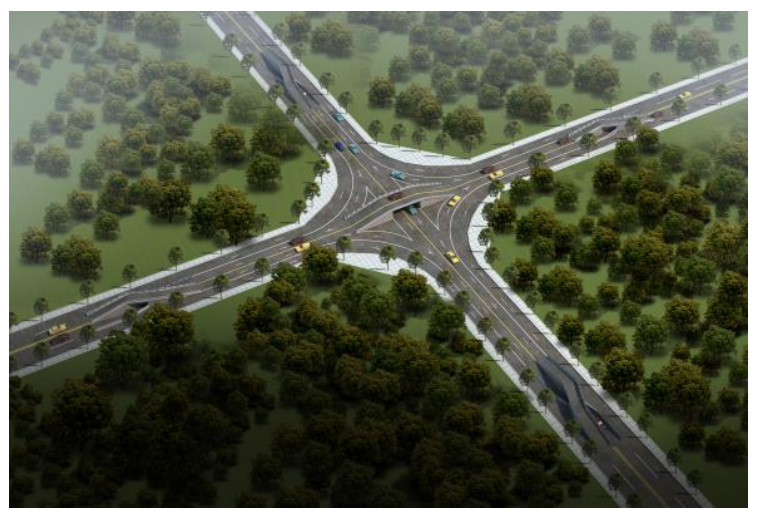

Fig. 8. Cross at the intersection to cancel the traffic light design.

\subsection{T-shaped intersection cancels the operation mechanism of traffic light design}

The T-shaped intersection is simpler than the intersection, except that the steering track of each of the inlet lanes is not exactly the same as the intersection except that it does not need to pass through the central overpass.

It is only necessary to operate according to the traffic without stagnation in the intersection. 

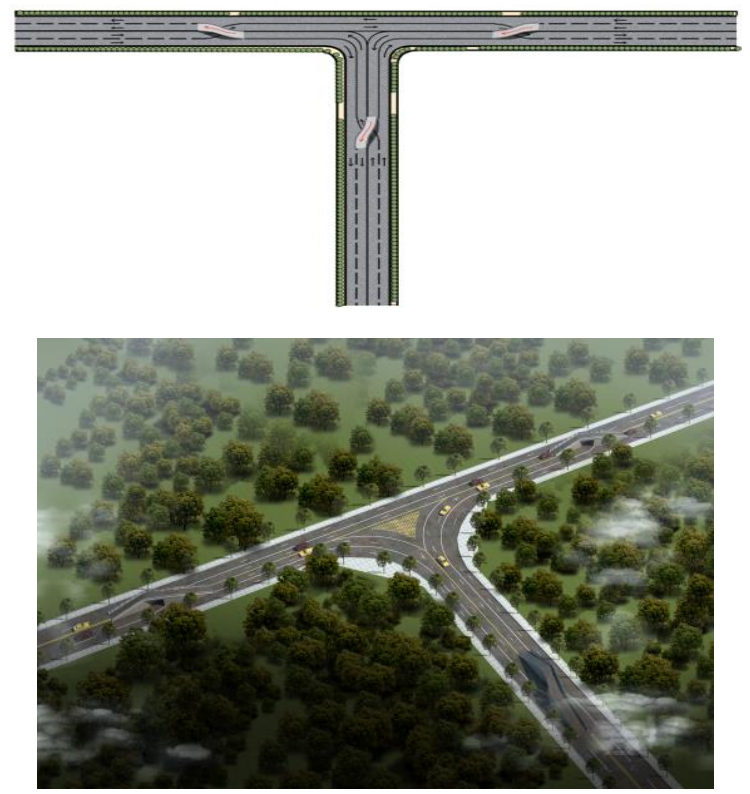

Fig. 9. T-shaped intersection cancels the operation mechanism of traffic light design.

\section{Discussion and Simulation analysis}

\subsection{Evaluation indicator}

According to the traffic characteristics of the intersection, and taking into account the fundamental role of road traffic in urban production and life, the following comprehensive evaluation elements are specifically proposed.

(a) Saturation: It is the ratio of the actual traffic volume $V$ to the traffic capacity at the intersection, reflecting the overall congestion of the intersection.

(b) Delay: Delay is a key operational performance indicator of intermittent traffic flow. It is the time loss caused by the vehicle passing through the intersection. It not only reflects the normal situation of the intersection, but also reflects the service level of the intersection. It is an important indicator for judging the performance of intersections.

(c) Capacity: The maximum number of vehicles passing through a section of a road in a certain road traffic environment. The unit is usually a car/hour.

(d) Average speed of vehicles passing through intersections: Due to more conflicts at intersections, traffic lights, traffic jams when there are many vehicles, etc., it often causes vehicles to wait while parking through the intersections, driving slowly, causing passage through the intersections. Long, the average speed of the vehicle passing through the intersection is that the average speed of the vehicle passing through the intersection is slow, so the average speed of the vehicle passing through the intersection is also an important comprehensive indicator for evaluating the capacity of the intersection.

\subsection{Non-stagnation design simulation solution.}


According to the building model of «Non-Roundabout design of cancel the intersection signal light on horizontal plane»and the principle of traffic diversion under the control of signal lights, the traffic simulation model of this design was established by using Vissim traffic simulation software, and the intersection of the intersections under the signal light was established by using Vissim traffic simulation software. Traffic simulation model. Then, the simulation model is run under the set conditions and the relevant evaluation indicators are obtained. According to the evaluation indicators, the feasibility and comprehensive capacity of the «Non-Roundabout design of cancel the intersection signal light on horizontal plane» [18].

1) Determination of data related to the simulation plan.

(a) No stagnant design related data:

a. The east-west direction lane is 391 meters long, and the north-south direction lane is 391 meters long.

b. Non-central small overpass long is 58 meters and slope $4.92 \%$.

c. The center overpass long is 60 meters and the slope $4.76 \%$.

$\mathrm{d}$. The overpass excavation depth is 2.5 meters and the overpass high is 2.5 meters .

e. Number of lanes: two-way eight lanes, left turn lane, right turn lane, straight line 1, straight line 2.

f. The lane wide is 3.5 meters .

g. Number of vehicles on the lane at each intersection: $<1>2500$ vehicles/h. $<2>7200$ vehicles/h.

h. Expected speed V : $60 \mathrm{~km} / \mathrm{h}$.

j. Data collection every ten minutes.

(b) Traffic light control intersection related data:

a. The east-west direction lane long, is 391 meters and the north-south direction lane is long 391 meters.

b. Number of lanes: two-way eight lanes, left turn lane, right turn lane, straight line 1 , straight line 2 .

c. The lane wide is 3.5 meters.

d. Number of vehicles on the lane at each intersection: 2,500 vehicles/h.

e. Expected speed V: $60 \mathrm{~km} / \mathrm{h}$.

f. Number of signal phases: 4 phases.

North and South straight green time: 60 seconds.

North and South turn left green time: 45 seconds.

Things go straight green time: 60 seconds.

Turn left and west to green light time: 45 seconds.

Yellow light time: 3 seconds.

Signal period: $\mathrm{T}=222$ seconds.

g. Data collection every ten minutes.

Note: (1-1) According to the previous survey data, at the morning and evening peak hours of the Binyue intersection in Hohhot, the maximum traffic volume of the import road is about 2,300 units/h, and the actual capacity is about 1,600 units/h.Therefore, it is practical to set the number of vehicles input to each entrance road to 2,500 vehicles/h.

(1-2) Because the theoretical minimum headway distance is $2 \mathrm{~s}$, when the minimum headway distance is $2 \mathrm{~s}$, the maximum number of vehicles passing through each lane within one hour is 1800 vehicles/ $h$, so the four-lane entrance The maximum traffic volume of the road is 7,200 vehicles/h. Therefore, the number of imported vehicles at each entrance is 7,200 vehicles/ $h$, which is the theoretical maximum. In the actual roads, due to the road conditions, the randomness of the driver and the vehicle arriving at the intersection, the minimum headway distance is less than 2 s. The traffic volume will not reach 7,200 
vehicles/h.Here we take the input traffic of each import road to 7,200 vehicles / $\mathrm{h}$ to test the ultimate capacity of this design [19].

2) Simulation model running display

(a) The intersection of the vehicles without stagnation design simulation program running show:

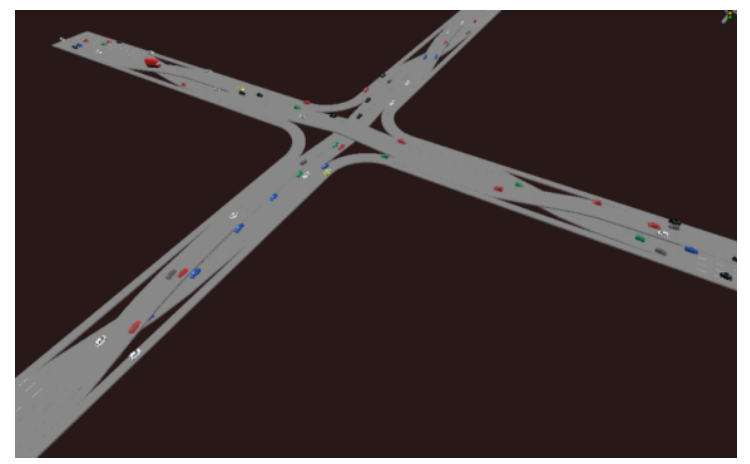

Fig. 10. Actual operation diagram of the vehicles without stagnation at the intersection (2500 vehicles/h).

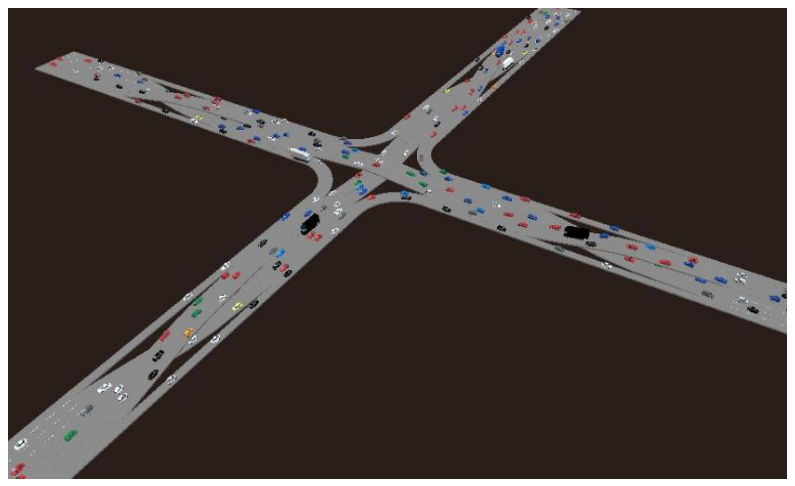

Fig. 11. Actual operation diagram of the vehicles without stagnation at the intersection (7200 vehicles/h).

(b) Signal light intersection simulation scheme operation display:

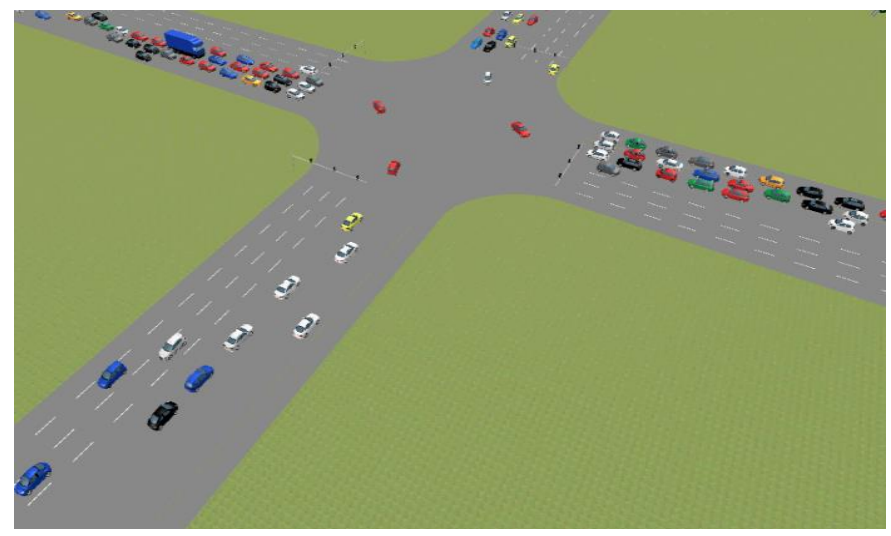

Fig. 12. Traffic Light Control Intersection Simulation Scheme Actual Operation Diagram. 


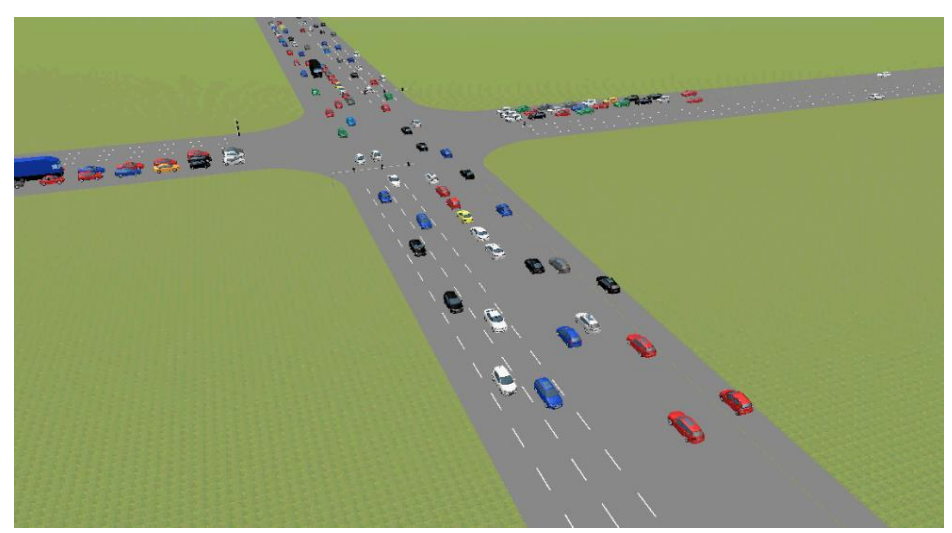

Fig. 13. Traffic light control intersection simulation scheme actual operation diagram.

3) Relevant evaluation indicators obtained from the simulation model operation.

Table 1. Relevant data indicators obtained from the operation of the traffic simulation simulation program.

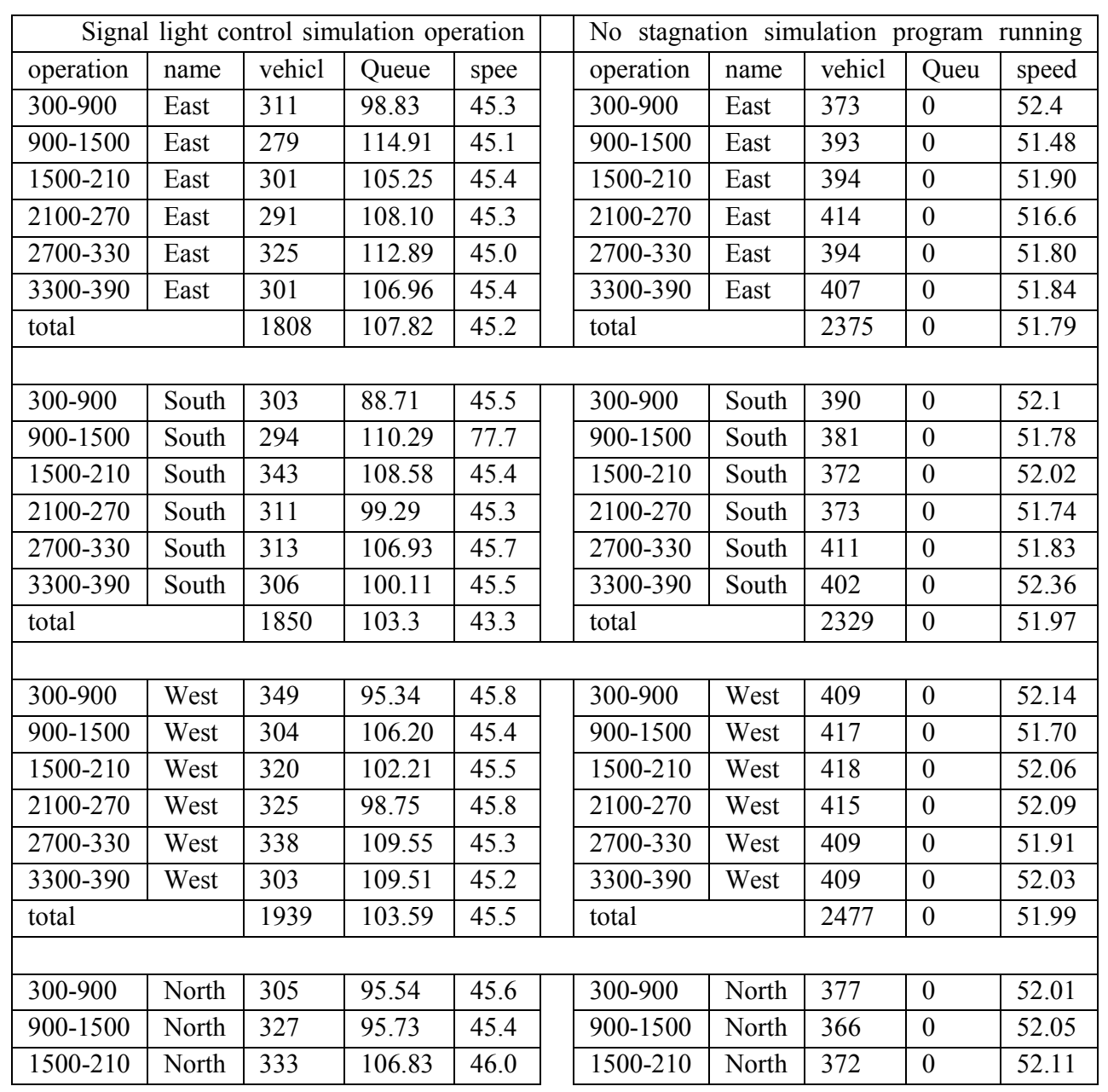




\begin{tabular}{|c|c|c|c|c|c|c|c|c|c|}
\hline $2100-270$ & North & 315 & 98.74 & 45.3 & $2100-270$ & North & 360 & 0 & 52.31 \\
\hline $2700-330$ & North & 325 & 95.71 & 46.1 & $2700-330$ & North & 420 & 0 & 52.19 \\
\hline $3300-390$ & North & 329 & 110.71 & 46.0 & $3300-390$ & North & 399 & 0 & 51.71 \\
\hline total & & 1934 & 100.54 & 45.7 & \multicolumn{2}{|l|}{ total } & 2294 & 0 & 52.06 \\
\hline
\end{tabular}

Table 2. Relevant data indicators obtained when the import road sets the limit traffic volume of 7,200 vehicles/h.

\begin{tabular}{|c|c|c|c|c|c|c|c|c|c|}
\hline \multicolumn{5}{|c|}{ Signal light control simulation operation } & \multicolumn{5}{|c|}{ No stagnation simulation program running } \\
\hline operation & name & vehicl & Queu & spee & operation & name & vehicl & Queu & spee \\
\hline $300-900$ & East & 1202 & 0.00 & 49.8 & $300-900$ & West & 1227 & 0.00 & 49.8 \\
\hline $900-1500$ & East & 1188 & 0.00 & 49.4 & $900-1500$ & West & 1157 & 0.00 & 50.0 \\
\hline $1500-210$ & East & 1202 & 0.00 & 50.0 & $1500-210$ & West & 1207 & 0.00 & 49.8 \\
\hline $2100-270$ & East & 1187 & 0.00 & 50.1 & $2100-270$ & West & 1174 & 0.00 & 49.7 \\
\hline $2700-330$ & East & 1188 & 0.00 & 49.0 & $2700-330$ & West & 1205 & 0.00 & 49.3 \\
\hline $3300-390$ & East & 12229 & 0.00 & 49.5 & $3300-390$ & West & 1189 & 0.00 & 49.5 \\
\hline \multicolumn{2}{|l|}{ total } & 7196 & & 49.6 & \multicolumn{2}{|l|}{ total } & 7159 & & 49.7 \\
\hline \\
\hline $300-900$ & South & 1218 & 0.00 & 49.9 & $300-900$ & North & 1172 & 0.00 & 50.0 \\
\hline $900-1500$ & South & 1222 & 0.00 & 49.6 & $900-1500$ & North & 1179 & 0.00 & 49.7 \\
\hline $1500-210$ & South & 1215 & 0.00 & 49.8 & $1500-210$ & North & 1200 & 0.00 & 49.9 \\
\hline $2100-270$ & South & 1121 & 0.00 & 50.2 & $2100-270$ & North & 1183 & 0.00 & 49.9 \\
\hline $2700-330$ & South & 1209 & 0.00 & 49.7 & $2700-330$ & North & 1197 & 0.00 & 49.3 \\
\hline $3300-390$ & South & 1265 & 0.00 & 49.4 & $3300-390$ & North & 1155 & 0.00 & 50.0 \\
\hline \multicolumn{2}{|l|}{ total } & 7250 & 0.00 & 49.8 & \multicolumn{2}{|l|}{ total } & 7086 & 0.00 & 49.8 \\
\hline
\end{tabular}

Table 3. Comparison of design schemes and original scheme evaluation indicators.

\begin{tabular}{|l|l|l|l|l|}
\hline \multicolumn{2}{|c|}{} & flow & Average delay & Average passing speed \\
\hline $\begin{array}{l}\text { East entrance } \\
\text { road }\end{array}$ & No stagnation & $\begin{array}{l}2375 \text { vehicles } \\
/ \mathrm{h}\end{array}$ & $0 \mathrm{~s}$ & $51.79 \mathrm{~km} / \mathrm{h}$ \\
\cline { 2 - 5 } & Original plan & $\begin{array}{l}1808 \text { vehicles } \\
/ \mathrm{h}\end{array}$ & $107.82 \mathrm{~s}$ & $45.29 \mathrm{~km} / \mathrm{h}$ \\
\hline $\begin{array}{l}\text { South Import } \\
\text { Road }\end{array}$ & No stagnation & $\begin{array}{l}2329 \text { vehicles } \\
/ \mathrm{h}\end{array}$ & $0 \mathrm{~s}$ & $51.97 \mathrm{~km} / \mathrm{h}$ \\
\cline { 2 - 5 } & Original plan & $\begin{array}{l}1850 \text { vehicles } \\
/ \mathrm{h}\end{array}$ & $103.3 \mathrm{~s}$ & $43.38 \mathrm{~km} / \mathrm{h}$ \\
\hline $\begin{array}{l}\text { West } \\
\text { Entrance } \\
\text { Road }\end{array}$ & No stagnation & $\begin{array}{l}2477 \text { vehicles } \\
/ \mathrm{h}\end{array}$ & $0 \mathrm{~s}$ & $51.99 \mathrm{~km} / \mathrm{h}$ \\
\cline { 2 - 5 } & Original plan & $\begin{array}{l}1939 \text { vehicles } \\
/ \mathrm{h}\end{array}$ & $103.59 \mathrm{~s}$ & $45.53 \mathrm{~km} / \mathrm{h}$ \\
\hline $\begin{array}{l}\text { North } \\
\text { entrance road }\end{array}$ & No stagnation & $\begin{array}{l}2294 \text { vehicles } \\
/ \mathrm{h}\end{array}$ & $0 \mathrm{~s}$ & $52.06 \mathrm{~km} / \mathrm{h}$ \\
\cline { 2 - 5 } & Original plan & $\begin{array}{l}1934 \text { vehicles } \\
/ \mathrm{h}\end{array}$ & $100.54 \mathrm{~s}$ & $45.77 \mathrm{~km} / \mathrm{h}$ \\
\hline
\end{tabular}




\subsection{Analysis of results}

Simulate the software to simulate the channelization design of the signal light at the two-dimensional intersection, and then analyze the data obtained by the simulation [20]. At the same time, we can evaluate the design using the formula without traffic light in intersection. Traffic volumes of intersection:

$$
Q_{h}=Q_{Z}+Q_{C}
$$

$Q_{z}=$ Road design traffic volumes

$Q_{c}=\frac{Q_{h} e^{-q a}}{1-e^{-q b}}$ (vehicles/hour)

q-main road traffic flow, a-minimum clearance, b-average headway distance of branch road.

1) Comparative analysis of delays:

From the comparison of the data in Table 3 above, when the traffic volume of 2,500 vehicles/h is set in each import road, the non-stagnation scheme is delayed to 0 in the whole process, The original design, controlled by traffic lights, had an average delay of more than $100 \mathrm{~s}$, and the vehicles lined up as they passed through the intersection. When the non-stagnation design scheme sets the limit traffic volume of 7,200 vehicles/h on each entrance road, the delay is still 0 (as shown in Table 2). Therefore, in the case of vehicle queue delay when crossing the intersection, the non-stagnation design truly reaches vehicle needn't to park and wait for the design purpose.

2) Traffic stream comparison analysis:

From the data comparison in Table 3 above, it can be seen that under the same traffic flow setting of 2,500 vehicles/h, the non-stagnation design scheme can realize that each import road passes around 2,400 vehicles per hour, and the traffic capacity of the entire intersection is in each about 9,600 vehicles per hour. In the original scheme controlled by red street lights, each entrance road can pass around 1,800 vehicles per hour, and the intersection capacity is about 7,200 vehicles per hour. According to the previous survey data, at the morning and evening peak hours of the Hohhot-Binyue intersection, the maximum traffic volume of the import road is about 2,300 vehicles $/ \mathrm{h}$, and the actual traffic capacity is about 1,600 vehicles/h. Therefore, compared with the actual intersection capacity in real life, compared with the simulation scheme under the control of traffic lights, the traffic capacity of the intersection non-stagnation design is absolutely superior.

3) Comparative analysis of average speed through intersections:

From the comparison of the data in Table 3 above, the non-stagnation design has no average waiting delay when the vehicle passes through the intersection, so the average speed of the vehicle when passing through the intersection is about $51 \mathrm{~km} / \mathrm{h}$. And the original scheme under the control of the traffic light is stopped, delay in waiting, the average speed of the vehicle as it passes through the intersection is $45 \mathrm{~km} / \mathrm{h}$ and around. In contrast, when the intersection traffic reaches 2,500 vehicles/h, the average speed of the vehicle with no stagnation design through the intersection is higher than that of the red street light control, which proves that the cancellation of the traffic light can greatly improve the traffic capacity of the intersection.

4) Saturation calculation and analysis verification of the design intersection without stagnation:

According to the saturation calculation formula [21]: $\mathrm{p}=\mathrm{v}$ (actual traffic volume) / $\mathrm{c}$ (pass capacity), the saturation of the design-free intersection can be calculated as: 
$\mathrm{p}=2300 / 7200=0.32$ (peak car at Binyue intersection) The flow is calculated based on the actual traffic volume)

After calculation, the saturation of the intersection without design is $p=0.32<0.6$. According to the specification, the intersection service level is a level when the intersection saturation is less than 0.6, and the level a service level indicates that the intersection is unblocked, and the vehicle is almost unnecessary. Waiting for parking, the vehicle receives the highest level of service.Therefore, the non-stagnation design can reach the highest level at the intersection service level, in line with the expected no-stagnation scenario.

\section{Conclusions}

This design is a channelized design scheme based on traffic engineering and transportation management theory to solve urban traffic congestion as a research purpose. It is a complete system with strong comprehensive service capability and strong enforceability. It can not only adapt to the complex and changing traffic situation, but also eradicate traffic congestion and adapt to the efficient and convenient traffic demand in the CBD area.

This design is not only a intersection-channelization system with super practicality, also a system with good innovative inventions. It has made innovative inventions in many ways. The representative innovations are as follows:

5) The design uses the channelization method to plan the urban CBD area, and realizes the function of the separation and traffice stream (flow) of the three-dimensional traffic space on the horizontal plane. The canalization design not only solves a series of traffic problems caused by traffic light control, but also obtains all interworking functions at the intersection, and solves the congestion problem in the CBD area well.

6) The straight line and the left turn traffic are separated in advance, and then the convergence theory is later.

Compared with the current straight-forward and right-turn combined driving and left-turning alone, the design can better share the straight traffic and the left-turn traffic on the road segment, and distribute the traffic flow at the intersection evenly, thus making the intersection road It is more fully adapted to the needs of various traffic flows in the CBD area.

7) The intertwined confluence area is dedicated to single-channel traffic, horizontal retrograde, and pipeline-type transportation. The intersection area is dedicated to single-passage, and the horizontally retrograde traffic organization completely separates the number of traffic conflicts. In addition, the traffic flow in the tubular organization intersection area forces the vehicles to travel sequentially, reducing the confluence and greatly increasing the driving efficiency of the intersection.

8) Separate semi-lowering and half-erecting calculation model for the position of interchanged piers, broadening the planning principle of separating the ramps and adding sections, and proposing the width function of the confluence bridge.

9) In combination with the situation of mixed driving between Chinese motor vehicles and non-motor vehicles, the implementation of segmented mixed traffic, the separation of segmented motor vehicles and non-motor vehicles, and the implementation of motor vehicles and non-machines in areas where the number of motor vehicles is too high. Separation of motor trains is based on the use of adjacent streets and lanes, and then the non-motor vehicles are collected in a specific lane. Finally, there is no stagnation and centralized traffic. In the area where the traffic volume is too small, motor vehicles and non-motor vehicles are mixed. 


\section{References}

1. T. Xiaojie, Research on Knowledge-Based Methodologies and System for Decision Making of Traffic Congesion Management (Southeast University, Jiangsu, 2005)

2. Z. Wei, Study on the Mechanism of Congestion and Diffusion of Intersection Groups and Its Control and Induction Cooperative Model (Jilin University, Jilin, 2013)

3. Li Xiaoshuai, Research on traffic organization optimization of large-scale intersections in cities (Beijing Jiaotong University, Beijing, 2009)

4. L. Weihong, Y. Weida, Urban Road Irregular Intersection Planning, Now Traffic Technology (Traffic Evening News, Beijing, 2009)

5. H. Shangqiu, Research on phase optimization and signal control method of single intersection (Zhejiang University of Technology, Zhejiang, 2011)

6. W. Yao, Optimization design and effect evaluation of plane signal intersections (Chang'an University, Chang'an, 2013)

7. J. Li, Research on traffic organization optimization of signalized intersections under mixed traffic flow (Chang'an University, Chang'an, 2006)

8. S. Chunfu, Principles of Traffic Planning (China Railway Publishing House, Beijing, 2015)

9. M. Liang, Research on some problems in the design of urban road intersections (Nanjing Forestry University, Nanjing, 2011)

10. L. Jiang, Traffic Flow Theory, Traffic Engineering (China Communications Press, Beijing, 2002)

11. H. Ping, Traffic flow characteristics of roundabouts (School of Transportation, Jilin University, Jilin, 2011)

12. S. Huijin, Study on the probability of mixed traffic conflicts at urban signalized intersections (School of Transportation, Beijing Jiaotong University, Beijing, 2008)

13. Y. Jingwei, Comparative analysis of traffic capacity calculation methods at signalized intersections, highway traffic technology (Chongqing Communications Commission, Chongqing, 2006)

14. W. Shibin, C. Weixiong, Traffic Standardization (Ministry of Communications, Beijing, 2010)

15. L. Meiling, Transportation Planning and Management (China Communications Press, Beijing, 2004)

16. K. Changjiu, Research on channelization method of traffic control intersections (School of Transportation, Beijing University of Technology, Beijing, 2006)

17. M. Rongguo, Y. Libo, Theory and Method of Traffic Engineering Design (China Communications Press, Beijing, 2002)

18. S. Feng, Y. Xiaoguang, Natural Science Journal of Tongji University 04(6), 06 (2009)

19. L. Yunhui, L. Lin, Q. Wenzhou, Journal of Highway and Motor Transport 74(1), 47 2017

20. L. Haitao, C. Zhi, Transportation Magazine 84(6), 45 (2016)

21. C. Xueyan, Journal of Natural Science of Jilin University 26(1), 63 (2012) 